\title{
ANÁLISIS LOGÍSTICO AGROCADENA DE ALGODÓN COLOMBIA EMPLEANDO DINÁMICA DE SISTEMAS
}

\section{LOGISTIC ANALYSIS COTTON AGROCHAIN COLOMBIA USING SYSTEM DYNAMICS}

\author{
Rafael Alfonso Toro Guzmán', Mauricio Becerra Fernández²,
}

\begin{abstract}
RESUMEN
Este estudio tiene como finalidad analizar la agrocadena del algodón en Colombia empleando dinámica de sistemas para identificar potenciales mejoras a nivel logístico y de tomas de decisiones, teniendo en cuenta los crecimientos y variables como área cosechada y producción del sector algodonero en Colombia. Los resultados mostraran un análisis propio actual del sector y sus escenarios a partir de variables como el precio, oferta, capacidades de producción, entre otras. Estos resultados brindaran un diagnóstico claro del sector actual en temas de producción anual y tiempos de producción con el fin de realizar un modelo que genere mejoras en las variables y la inclusión políticas y prácticas que impulsen la cadena del algodón en términos logísticos y económicos. Los escenarios planteados en el estudio muestran una alta correlación por los datos otorgados por los entes interesados en este sector, indicando que el modelo de dinámica de sistemas resulta ser una herramienta idónea, en la creación de escenarios principalmente a la facilidad de interacción e interpretación de los resultados.
\end{abstract}

Palabras Clave: Algodón, Dinámica de sistemas, Agro Cadena, Logística

\section{ABSTRACT}

The purpose of this study is to analyze the cotton agro-chain in Colombia using systems dynamics to identify potential logistic and decision-making improvements, taking into account the growth and variables as a harvested area and production of the cotton sector in Colombia. The results will show a current own analysis of the sector and its scenarios based on variables such as price, supply, production capacities, among others. These results will provide a clear diagnosis of the current sector in terms of annual production and production times in order to create a model that generates improvements in the variables and the inclusion of policies and

\footnotetext{
${ }^{1}$ Maestría en Ingeniería Industrial, Facultad de Ingeniería, Universidad Católica de Colombia, Bogotá, Colombia. orcid.org/0000-0002-5054-8520

${ }^{2}$ Grupo de Investigación en Producción (GIP) y Programa de Ingeniería Industrial, Facultad de Ingeniería, Universidad Católica de Colombia, Bogotá, Colombia. orcid.org/0000-0003-1060-2198
} 
practices that drive the cotton chain in logistic and economic terms. The scenarios proposed in the study show a high correlation for the data provided by the entities interested in this sector, indicating that the system dynamics model turns out to be an ideal tool, in the creation of scenarios mainly to the ease of interaction and interpretation of the results.

Keywords: Cotton, Systems dynamics, Agro Chain, Logistics.

\section{INTRODUCCIÓN}

La cadena del algodón tiene una gran trayectoria y dinamismo en la industria colombiana. Esto se ha consolidado como un sector fuerte a nivel de producción, generación de empleo, su desarrollo económico orientado a la utilización de nuevas tecnologías, a la potencialidad de inversiones y al aumento de las exportaciones de productos acabados. El sector ha presentado en los últimos años un crecimiento debido a la cantidad y calidad de productos terminados, principalmente hilados, tejidos para la confección de prendas de vestir y artículos para el hogar, el cultivo del algodón tiene su importancia económica no solo por sus varias décadas de llevarse ejerciendo como actividad, sino principalmente por la influencia que tiene el sector en los municipios de la región interior y costa que es donde se concentra la mayor área de cultivo sembrada. Según Conalgodon (2015), se sembraron en las regiones interior y costa alrededor de 40000 ha para el año 2015 que significaron en la producción unas 32000 (T) de fibra de algodón (Figura 1). La tendencia en la relación hectáreas sembradas y producción generan un rendimiento creciente en ha/T, pero se evidencia que ha disminuido la cosecha en estos últimos años, por consiguiente la producción disminuye.

De acuerdo con el DANE (2007) la industria textil y de confecciones representa el 7,33\% de la producción industrial nacional. Para el año 2006 genero un total de 132 mil empleos y obtuvo una producción superior a los 3040 billones de dólares, siendo fabricación de prenda de vestir, excepto pieles y tejeduría de productos textiles, los productos que en mayor cantidad se producen en el país.

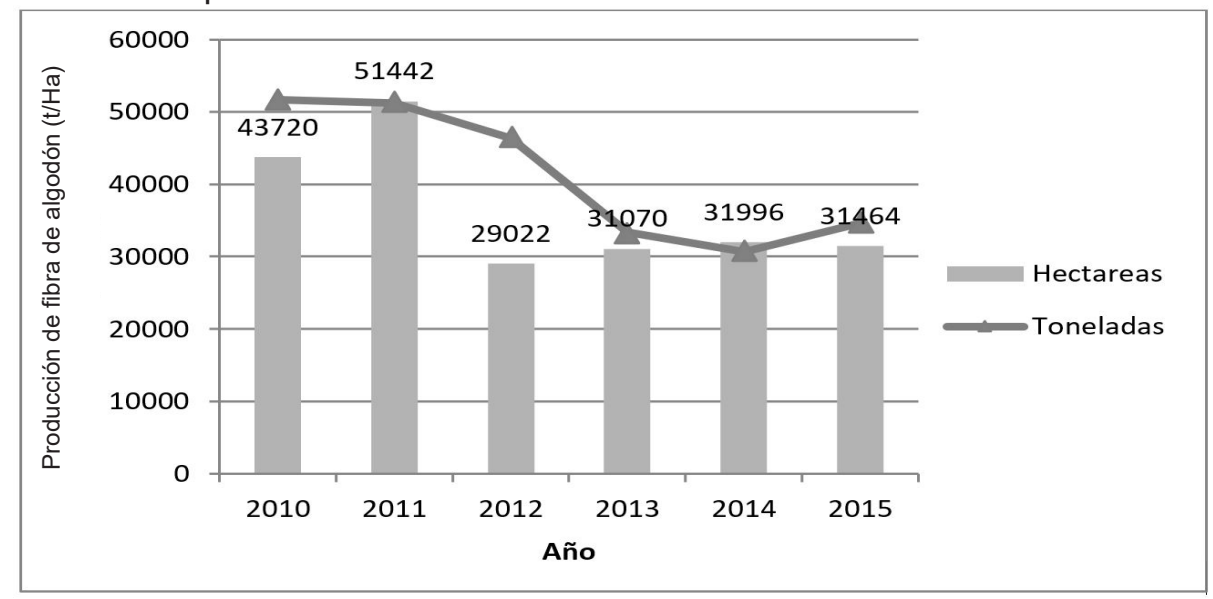

Figura 1. Evolución de la fibra de algodón toneladas/hectáreas (2010-2015).

Fuente: Conalgodon, 2015.

\section{Descripción del cultivo de algodón}

El algodón es el producto agrícola no alimentario más importante del mundo y su cultivo es 
de los más antiguos. En un principio la palabra algodón significaba "un tejido fino". La planta de algodón posee un tallo erecto y con ramificación regular, las hojas son pecioladas, de un color verde intenso, grandes y con los márgenes lobulados, están provistas de brácteas. Las flores son dialipétalas, grandes, solitarias y penduladas. La corola está formada por un haz de estambres que rodean el pistilo. Se trata de una planta autógama, aunque algunas flores abren antes de la fecundación, produciéndose semillas híbridas. El fruto es una cápsula en forma ovoide con tres a cinco carpelos, que tienen seis a diez semillas cada uno, es de color verde durante su desarrollo y oscuro en el proceso de maduración. Las células epidérmicas de las semillas constituyen la fibra llamada algodón según Conabio (2008).

\section{Producción de fibra de algodón en Colombia}

En Colombia, el algodón es un producto agrícola de ciclo semestral, logrando dos temporadas algodoneras en el año. La temporada del "Interior" inicia sus siembras a comienzos del año y cosecha su producción entre julio y octubre de cada año, en los departamentos de Cundinamarca, Tolima y Valle del Cauca. La temporada "Costa" inicia sus siembras a mediados de cada año y recolecta la producción entre diciembre y marzo del siguiente año, tradicionalmente en los departamentos de Atlántico, Bolívar, Cesar, Córdoba, Guajira, Magdalena, Sucre y Vichada y es la temporada que aporta el mayor porcentaje de la producción nacional. Se relaciona el volumen producido de fibra de algodón a nivel regional para el año 2012; la región costa cuenta con la mayor participación dentro del total producido, $78,83 \%$ frente a las $21,17 \%$ de la región interior, respectivamente en el transcurso de los años la región costa siempre ha tenido mayor influencia en la producción de fibra de algodón en Colombia, seqún la Superintendencia de Industria v Comercio (2010) (Fiqura 2).

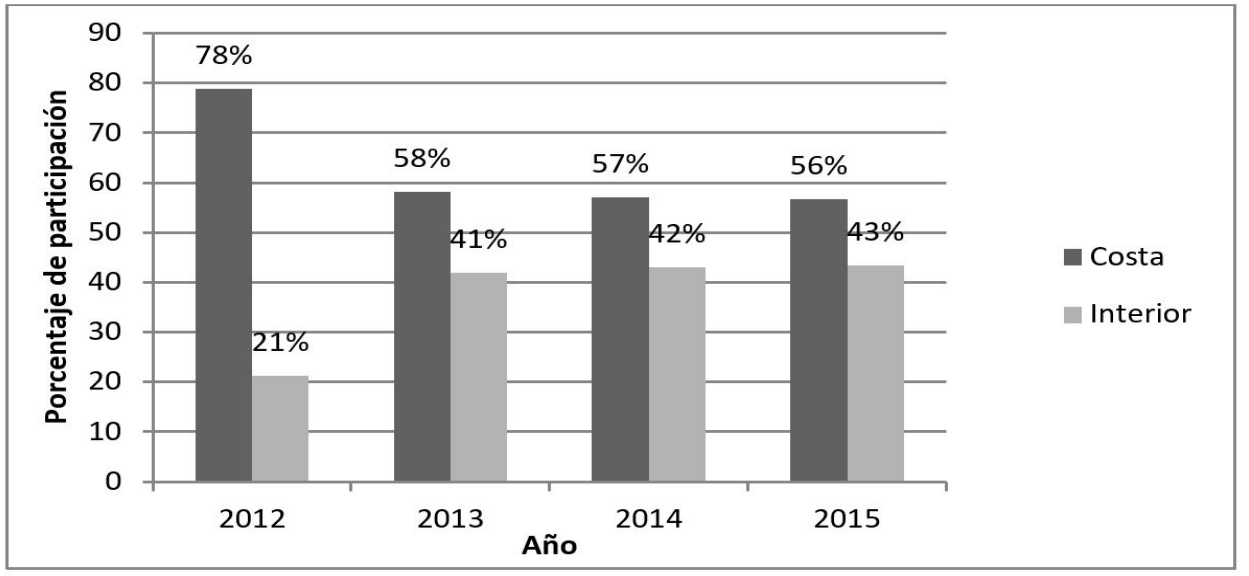

Figura 2. Producción de fibra de algodón por regiones en Colombia en \% 2012-2015 Fuente: SIC, 2010

En la Figura 2, se puede observar que los años de mayor producción corresponden a 2012 y 2013. A partir del año 2012 y hasta 2015, la producción de fibra mostró una tendencia decreciente, cuyo descenso promedio anual fue del 13\%, alcanzando el menor nivel del periodo bajo análisis en el año 2012 fue de 29829 (T). En el año 2013 se evidenció cierta recuperación, se produjeron 32000 (T) de fibra de algodón, esto es, 2500 más que en el año anterior, que representó un incremento del $20 \%$. Sin embargo, esta leve recuperación en la producción puede verse contraída puesto que el número de hectáreas cosechadas pasó de 44334 ( $\mathrm{T}$ ) en el 2011 a 38952 ( $\mathrm{T}$ ) en el 2012. Es decir, se redujo el área cosechada en un 12\% lo que puede implicar que la producción de fibra también haya decaído en una proporción significativa para este año. 
De acuerdo con el Instituto Colombiano Agropecuario ICA (2010), cabe mencionar que la producción de algodón y fibra de algodón se encuentra regulada por el ICA, quien determina para cada temporada del año las fechas dentro de las cuales se admite la compra y venta de semilla, siembra, destrucción de socas y el registro de los agricultores. El registro de los agricultores debe hacerse a través de su agremiación o asociación, indicando el lote sembrado. Igualmente, el ICA define la fecha máxima de recibo de algodón semilla por parte de las desmotadoras (el proceso de desmoste se conoce como proceso de limpieza y clasificación del algodón).

\section{Las cadenas de suministro y la dinámica de sistemas}

A partir de los años 50 se desarrolla la dinámica de sistemas y se han construido muchos modelos siguiendo los principios de esta disciplina, en áreas tan diversas como las ciencias sociales, económicas, ambientales, administrativas y biológicas. A continuación, se hace una reseña de las investigaciones que han contribuido al desarrollo del SupplyChain Management (SCM, por sus sigla en inglés) o Administración de la Cadena de Abastecimientos.

Con la aparición de los computadores digitales y la dinámica de sistemas el profesor Jay W Forrester (1971) es el primero en formalizar la dinámica de sistemas en problemas de la administración de la cadena de abastecimiento. En el contexto Colombiano, Alarcón (2016) ha indicado que para el mejoramiento de la cadena, se hace necesario la inversión de los eslabones de la cadena para dar respuesta sincronizada y tener mejores niveles de servicio. Herrera y Orjuela (2014) tienen como propuesta la inclusión del efecto de trazabilidad de tecnologías para las cadenas de suministro de frutas y sus mejorías en la capacidad y calidad de los productos. Arroyo et al. (2012) utilizando una metodología de la dinámica de sistemas han simulado cómo la tasa de productos retornados por los individuos y la cantidad de computadoras recuperadas en una cadena de logística inversa de lazo abierto varía bajo distintos escenarios. Para Martínez et al. (2013) por medio de la aplicación equilibrada de las prácticas de gestión de conocimiento en la dimensión estratégica y en la dimensión funcional, se consigue incrementar la producción a través de la estabilización del sistema. La propuesta la gestión del conocimiento afecta positivamente las cadenas de suministros.

Otros casos de relación cadena de suministros y dinámica de sistemas conducen un ejemplo esencial en el estudio de la dinámica; según Sterman (1989) con el "Juego de la Cerveza" conduce un experimento para simular el manejo de la producción y distribución industrial, en el que se presentan varios actores, realimentaciones y retardos a lo largo de la línea de abastecimiento. En el juego se observa cómo el sistema exhibe tres comportamientos: oscilación, amplificación de las órdenes y retrasos en la cadena. Todo esto se debe a la poca percepción de realimentación, aunque en la vida real es importante tener en cuenta que los gerentes tienen acceso a más información de la que está disponible en el experimento. En el juego de la cerveza las oscilaciones se deben a que las reglas de decisión no tienen en cuenta los retrasos de materiales e información que hay entre el momento en que se pone la orden y cuando se reciben los materiales, además, explica el razonamiento utilizado por las personas para la toma de decisiones.

Diawati y Cakravastia (1999) describen un modelo de dinámica de sistemas que permite localizar cuellos de botella potenciales y pronosticar el desempeño logístico de una industria en particular. Definen el desempeño logístico con tres indicadores: calidad del producto, costo, y tiempo de entrega.

Tradicionalmente, el foco de atención se dirige a controlar el flujo de bienes físicos y de información; sin embargo, estudios indican que descuidar el flujo financiero puede causar 
problemas aún mayores que los otros dos, y reducir el desempeño logístico. Angerhofer y Angelides (2000) proponen que el modelo en dinámica de sistemas debe incorporar los tres flujos: físicos, de información y financieros, según

Holweg y Bicheno (2002) aplican Lean Leap Logistics Game sobre simulación de la cadena de abastecimientos y se refieren al juego de Lean Logistics, en el cual los autores expresan que en el juego de la cerveza se han encontrado varias limitaciones: es un juego enfocado solamente a la distribución y no toma en cuenta la transformación del producto; no tiene suficientes etapas que simulen la cadena de abastecimientos y no se encuentran características particulares del proceso de producción como los tiempos de preparación, cambios de referencia, problemas de calidad, etc. Es un juego que cuenta con capacidad ilimitada mientras que en la práctica se ha encontrado que ésta es una de las mayores restricciones. En general, el juego de la cerveza se ha criticado porque no tiene en cuenta la capacidad de producción y por ser un modelo limitado de la cadena de abastecimiento. Por lo anterior, los autores modelaron la cadena de una manera más participativa, teniendo en cuenta todas las características reales consideradas en "Lean Leap Logistics Game".

\section{METODOLOGÍA}

El modelo planteado de dinámica de sistemas de este artículo se realizó empleando el software Vensim versión 6.4. Soportado para el análisis de información en otros software tales como Microsoft Access, Microsoft Excel, StatFit, SPSS, a partir de información suministrada por los gremios y las entidades privadas del sector algodonero colombiano. La metodología parte de los datos iníciales de las variables a tener en cuenta en la cadena del algodón, pasando por el análisis de la variables de entrada del modelo y finalizando con la formulación de la cadena del algodón. (Figura 3)

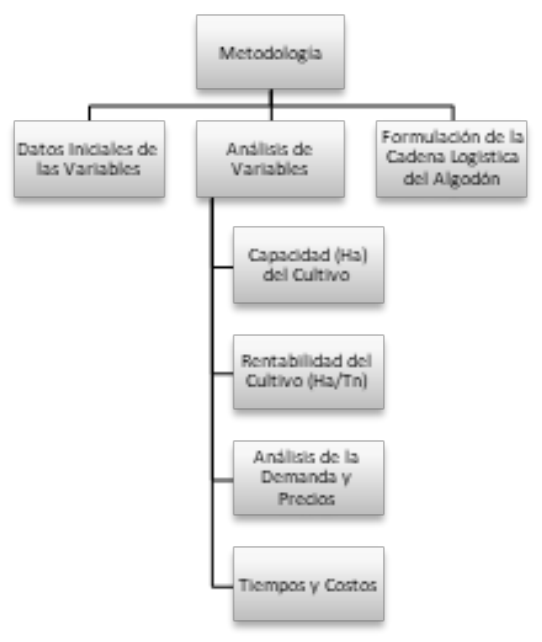

Figura 3. Metodología desarrollada

\section{Datos iníciales de las variables}

Al iniciar la investigación, se estableció como punto de partida la recolección de datos entregados mensual, semestral y anual por los gremios encargados de la medición como Conalgodon, gremios regionales y estadísticas nacionales por parte del DANE, al igual se recogieron datos en trabajo de campo con visitas a los cultivadores de algodón con el fin de verificar 
sus prácticas de sembrado y cosecha, identificando distintas variables de estudio, dentro de las visitas se recogieron datos en las desmotadoras industriales, esta visita se realizó con el fin de verificar los procesos después de la cosecha del algodón. Estos datos se tabularon para un mejor manejo en la herramienta Vensim, para realizar la corrida en el modelo.

\section{Análisis de las variables}

\section{Capacidad (ha) del cultivo}

Para el análisis de la Capacidad Hectárea del cultivo se tomaron los registros históricos de las bases de datos suministradas por Conalgodon, en las cuales se manejan en Microsoft Excel, obteniendo una tabla histórica de los últimos años por las regiones más representativas del algodón.

Posteriormente se analizó la variable, estableciendo tablas de tiempos de acuerdo a lo observado en el trabajo de campo realizado, con el fin de realizar las comparaciones con los datos suministrados por Conalgodon.

\section{Rentabilidad del cultivo}

Para el análisis de la rentabilidad del cultivo se tomaron los datos recogidos históricamente por Conalgodon año tras año, por lo que se generó una muestra promedio de la rentabilidad del cultivo de acuerdo a la época del año, y teniendo en cuenta la región de cultivo, para lo cual se generó un comparativo entre las regiones cultivadoras en el país.

\section{Análisis de la demanda y precios}

El análisis de estas variables se centró en los costos requeridos para obtener el producto final y su comportamiento a través de los eslabones productivos, los precios se tomaron de las bases de datos anuales por Conalgodon, que por medio de las agremiaciones regionales del algodón se tiene un estimado del valor final del producto, así como la demanda nacional e internacional, a través de organismos internacionales como el Banco Mundial e investigaciones en los últimos años.

\section{Tiempos y costos}

Para el análisis de tiempos y costos dentro del modelo al igual que la demanda y precios, los datos históricos se tomaron de las bases de Conalgodon y las agremiaciones que trabajan en conjunto en el sector del algodón. Esta variable es de importancia debido a que la investigación busca disminuir los tiempos entre eslabones, para lo cual se realizó cuadros de tiempos entre eslabón para determinar un promedio y así obtener mejores resultados.

\section{Formulación de la cadena logística del algodón}

La formulación de la cadena logística del algodón, busco potenciar y mantener la cadena del algodón dentro del mercado nacional, como pilar fundamental en la economía agrícola la cual ha permanecido por varias décadas evolucionando en Colombia y a nivel mundial como una de las cadenas más robustas.

La formulación se realizó a partir de las variables anteriormente escritas, para lo cual se utilizara la herramienta de Software Vensim, para iniciar algunos escenarios en busca de encontrar, el mejor prospecto para el estudio de la cadena y algunas decisiones puntuales dentro de la cadena que permitan la mejora y desarrollo dentro de la misma, reduciendo tiempos y costos, y demás factores que disminuyan la productividad de la cadena del algodón.

De acuerdo a la simulación planteada en el modelo de Vensim, se crearon varios escenarios validos dentro de las variables tenidas en cuenta en la cadena, los cuales se establecieron 
por datos reales, otorgados por los entes y agremiaciones inscritas en el sector del algodón a nivel nacional. Desde este punto de partida se observó varios puntos, en los cuales se puede tener en cuenta para la mejora y en otros donde las variables se encuentran en niveles altos tanto a nivel nacional como internacional. Algunas variables además fueron tenidas en cuenta a medida que la investigación tomo camino, esto debido a que los datos otorgados brinda información certera y precisa en el momento de la ejecución, pero se realizaron varios ajustes de acuerdo al manejo del trabajo de campo de la investigación, concluyendo factores que nos acercaron mucho más al trabajo esperado.

\section{DESARROLLO DEL MODELO}

El desarrollo del modelo se fundamentó en las etapas propuestas por Sterman para los modelos dinámicos, iniciando por la articulación del problema y finalizando en el análisis de los resultados del modelo. (Figura 4 )

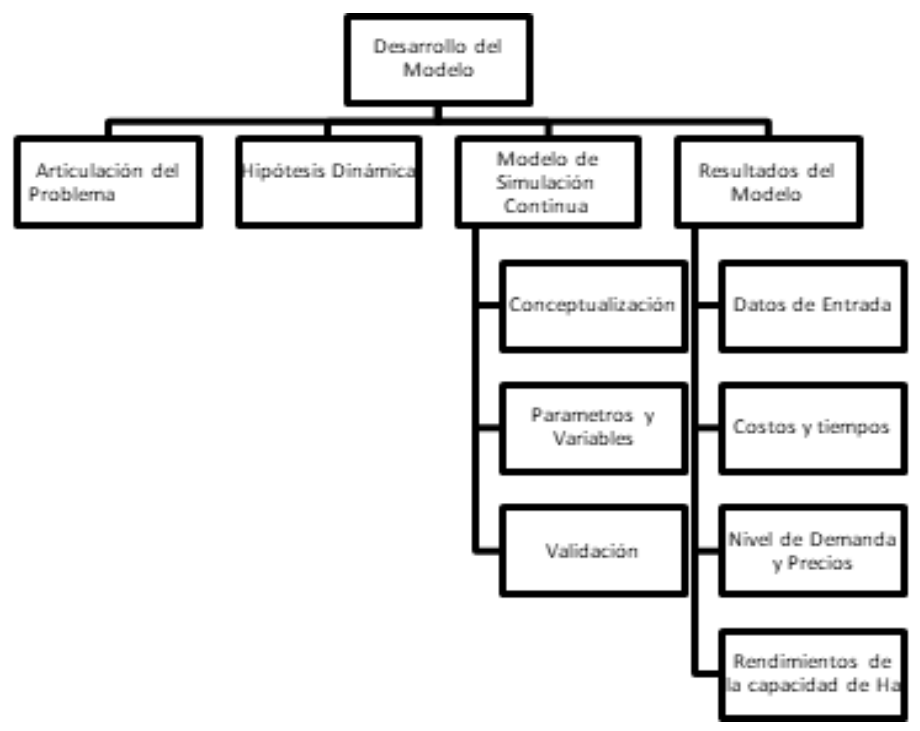

Figura 4. Estructura del Desarrollo del Modelo

Fuente: Elaboración propia.

\section{Articulación del problema}

Se buscó determinar los aspectos importantes a tener en cuenta en el Diseño Logístico Para La Reducción De Tiempos Improductivos En La Cadena De Suministro Algodón-Textil a nivel nacional, debido que para mejorar la competitividad de la cadena, se hace necesario el desarrollo de la infraestructura logística, tomando en consideración los ejes estratégicos identificados en el Plan de Desarrollo Nacional "Prosperidad para Todos "y el documentos realizados por el Departamento Nacional de Planeación "Visión Colombia Segundo Centenario".

De este modo se estudiaron diferentes parámetros como la políticas nacionales que se necesitan para implantar un diseño o logístico en la región, las matrices de origen-destino de cadena de suministro (producción, aprovisionamiento y distribución de los productos) de la cadena de suministros analizada, el diagrama de flujos con tiempos, la infraestructura y normatividad para la agro cadenas productiva, así como las posibles plataformas logísticas que 
se pueden implementar en el componente nacional.

Cabe señalar que la cadena de suministro analizada se escogió a partir de criterios como el precio, el área, total de producción y aceptación de mercados extranjeros con el fin de incrementar su índice de competitividad para que genere un crecimiento conjunto.

\section{Hipótesis dinámica}

Con el modelo del diseño logístico para la agrocadena del algodón de esta investigación empleado la herramienta de dinámica de sistemas, se buscó contrastar la hipótesis dinámica la cual considera que con la producción generada dentro de la cadena, se cumple con la demanda actual, de acuerdo a los recursos existentes.

\section{Conceptualización del modelo}

La idea original fue construir un modelo de dinámica de sistemas que simule los comportamientos en los procesos logísticos de la cadena del algodón, desarrollando un método que mejore la comprensión de las relaciones entre causa y efecto de la cadena; y que permita un mejor entendimiento entre las variables que afectan toda la cadena del algodón, obteniendo mejores resultados desde la capacidad para tomar decisiones, así como el mejoramiento interno de la cadena en términos de tiempos y costos, desarrollando mediante el modelo una mejor forma para entender este sistema logístico como lo es la cadena del algodón.

Es importante mencionar que el modelo realizado no sirve como herramienta de estimación a pesar de la creación de múltiples escenarios que evidencian mejorías en la cadena, la aplicación del modelo está diseñada para la toma de decisiones entre los componentes del sistema. (Figura 5).
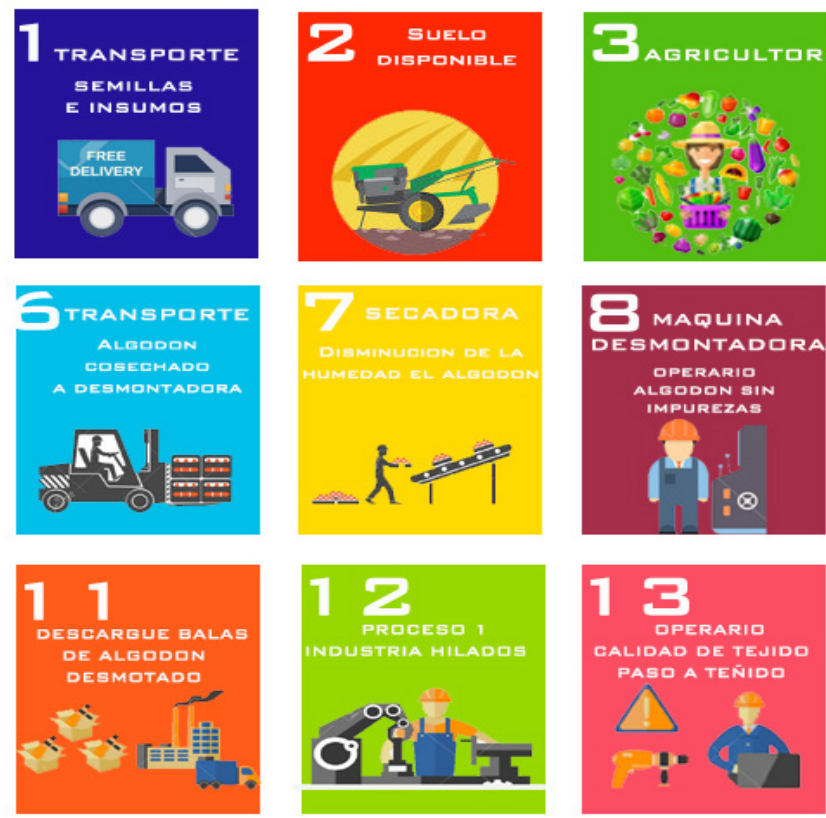
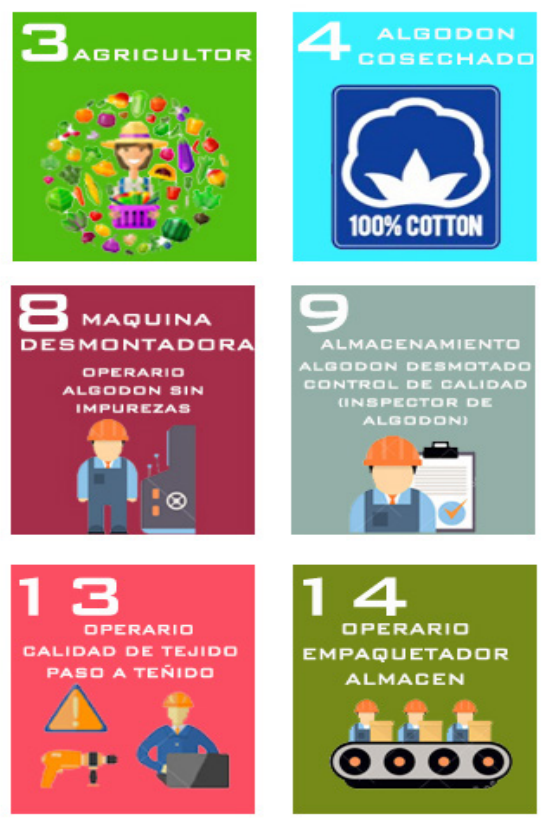
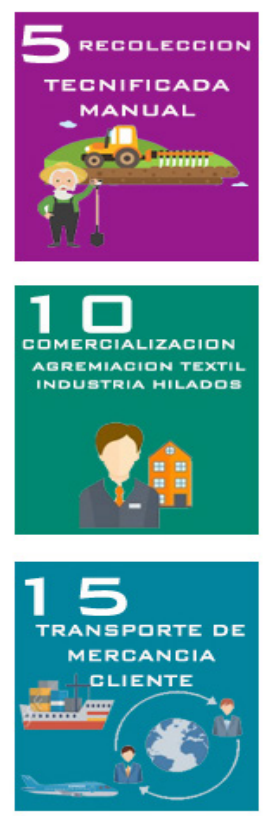

Figura 5. Conceptualización del Modelo. (Cadena Logística del Algodón) Fuente: Elaboración propia.

El modelo elaborado cuenta con los parámetros, variables de nivel, variables de flujo y variables auxiliares que se especifican a continuación. (Tabla 1). 
Los parámetros, su descripción y unidad de medida se muestran así:

Tabla 1. Parámetros del modelo (Cadena Logística del Algodón)

\begin{tabular}{|c|c|c|c|}
\hline $\mathbf{N}$ & Nombre & Descripción & Unidad de Medida \\
\hline 1 & Hectárea & Hectárea Cultivo & ha \\
\hline 2 & Ocupación Cosecha & Suelo Disponible / Porcentaje Uso Suelo & ha/día \\
\hline 3 & Aumento de Suelo & Nuevos Suelos Adaptados & ha/día \\
\hline 4 & Cultivo de Algodón & Suelo Disponible / Porcentaje Uso & ha/día \\
\hline 5 & Tasa de Cosecha & Suelo Sembrado / Rendimiento Suelo & t/día \\
\hline 6 & Rendimiento Suelo & Rendimiento del Suelo & t(ha*día) \\
\hline 7 & Actividad de Migración & Actividad de Migración del Suelo a Otro & Adimensional \\
\hline 8 & Porcentaje de Migración & Porcentaje de Migración del Suelo a otro & Adimensional \\
\hline 9 & Transporte Algodón & $\begin{array}{l}\text { Cosecha del Algodón / Días Transporte Cosecha a } \\
\text { Planta }\end{array}$ & t/día \\
\hline 10 & Rendimiento de Limpieza & Rendimiento de Limpieza & Adimensional \\
\hline 11 & Tasa de Desmote & $\begin{array}{l}\text { Inventario Algodón Cosechado / Capacidad Desmo- } \\
\text { tadora y Rendimiento de Limpieza }\end{array}$ & t/día \\
\hline 12 & $\begin{array}{l}\text { Transporte Algodón Desmo- } \\
\text { tado }\end{array}$ & Inventario Algodón Desmotado / Días de Transporte & t/día \\
\hline 13 & Rendimiento Hilandería & Rendimiento Hilandería & Adimensional \\
\hline 14 & Capacidad Desmotadora & Capacidad Desmotadora & día \\
\hline 15 & Capacidad Hilandería & Capacidad Hilandería & día \\
\hline 16 & Tasa Hilatura & $\begin{array}{l}\text { Inventario Algodón Desmotado /Capacidad Hilande- } \\
\text { ría y Rendimiento Hilandería }\end{array}$ & t/día \\
\hline 17 & Días Transporte Cos a Plan & Días de Transporte & día \\
\hline 18 & Días Transporte Des a Hilan & Días de Transporte & día \\
\hline
\end{tabular}

Fuente: Elaboración propia.

\section{Validación del comportamiento del modelo}

El modelo (Figura 6) presento variaciones de acuerdo a los escenarios planificados, de acuerdo a los datos y variables a los que se simularon dentro de los años 2015 y 2016 basados en las estadísticas de la entidad Conalgodon, quien se encarga de tener las cifras más actualizadas de los gremios que acompañan la agrocadena del algodón.

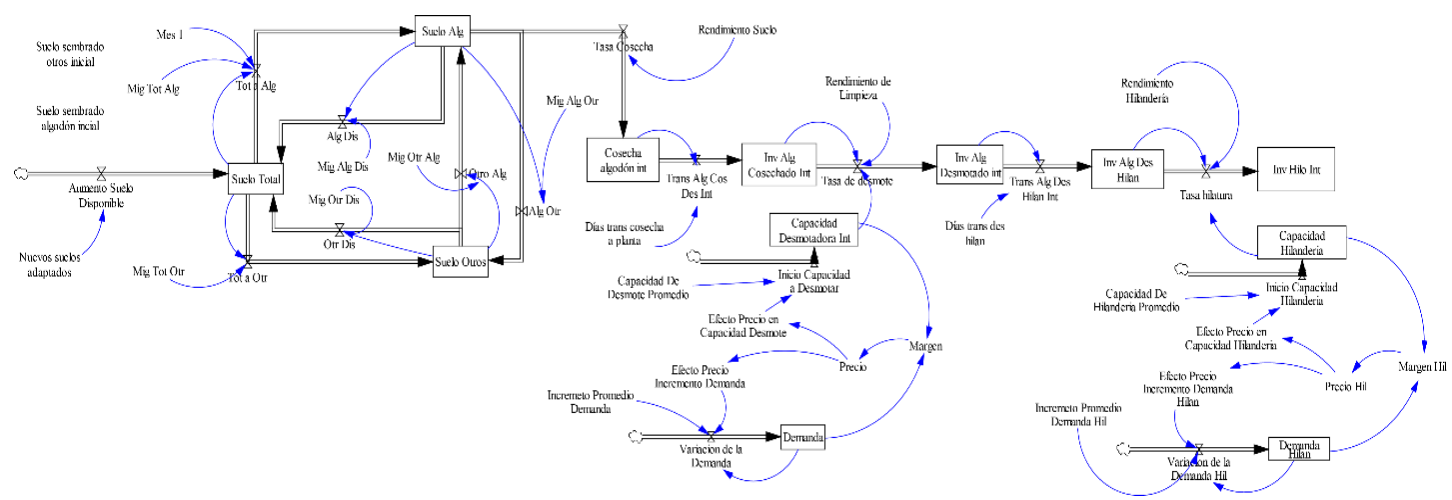

Figura 6. Diagrama de Forrester contemplado para la agrocadena del algodón en Colombia Fuente: Elaboración propia 


\section{RESULTADOS}

\section{Estructura del modelo}

Para la construcción del modelo se consideraron como datos de entrada la disponibilidad del suelo utilizado en actividades agrícolas y, el uso del suelo de la cadena de algodón en las regiones donde este se cultiva.

Se tomaron en cuenta el número de hectáreas totales aptas para el cultivo de otras cadenas agrícolas y dentro del modelo se analizaron, la migración del suelo entre las otras cadenas agrícolas y la movilidad, en el uso de suelo disponible para la cadena del algodón. (Figura 7).

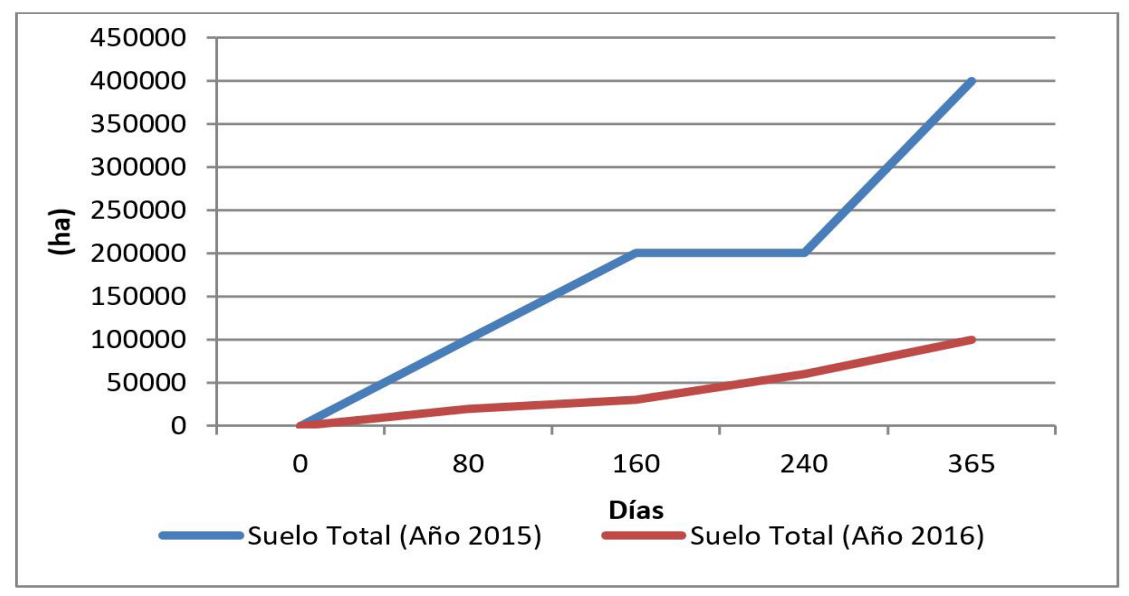

Figura 7. Total hectáreas por cultivo

Se estableció dentro del modelo la validación de los escenarios, en los años 2015-2016, mostrando un comportamiento en cuanto a crecimientos similares a la última década del sector. Las tablas 2 y 3 muestran la configuración que se consideró, de los parámetros y sus valores para la validación de los escenarios planteados para la agrocadena del algodón en Colombia;

Tabla 2. Uso del suelo/número de hectáreas

\begin{tabular}{|l|c|}
\hline \multicolumn{1}{|c|}{ Uso del Suelo } & Numero de ha \\
\hline Suelo Disponible Otros & 4000000 \\
\hline Suelo Algodón Inicial & 80000 \\
\hline Suelos Adaptados al Algodón & 5000 \\
\hline
\end{tabular}

Se consideraron los niveles de movilidad de los suelos de acuerdo a las demoras definidas al tiempo requerido de adaptación, como su muestra Tabla 3:

Tabla 3. Niveles de movilidad

\begin{tabular}{|l|c|c|}
\hline Demora de/a & Suelo Algodón & Suelo Otros \\
\hline Suelo Algodón & 0 & 0,08 \\
\hline Suelo Otros & 0,2 & 0 \\
\hline
\end{tabular}


El modelo desarrollado se evaluó a partir de dos escenarios que combinan los siguientes elementos:

- E1: Movilidad del Suelo Otros a Suelo Algodón.

- E2: Movilidad del Suelo Algodón a Otros.

- E3: Aumento de la Capacidad de Hilandería Promedio.

- E4: Disminución de la Capacidad de Hilandería Promedio.

Las políticas de movilidad permiten que el suelo usado en un sector se mueva al otro, considerando las demoras comentadas anteriormente. Los aumentos y disminución de la capacidad se deben a políticas de mercado externa que varían el comportamiento.

Los escenarios planteados se muestran en la tabla 4 :

Tabla 4. Escenarios contemplados para el modelo

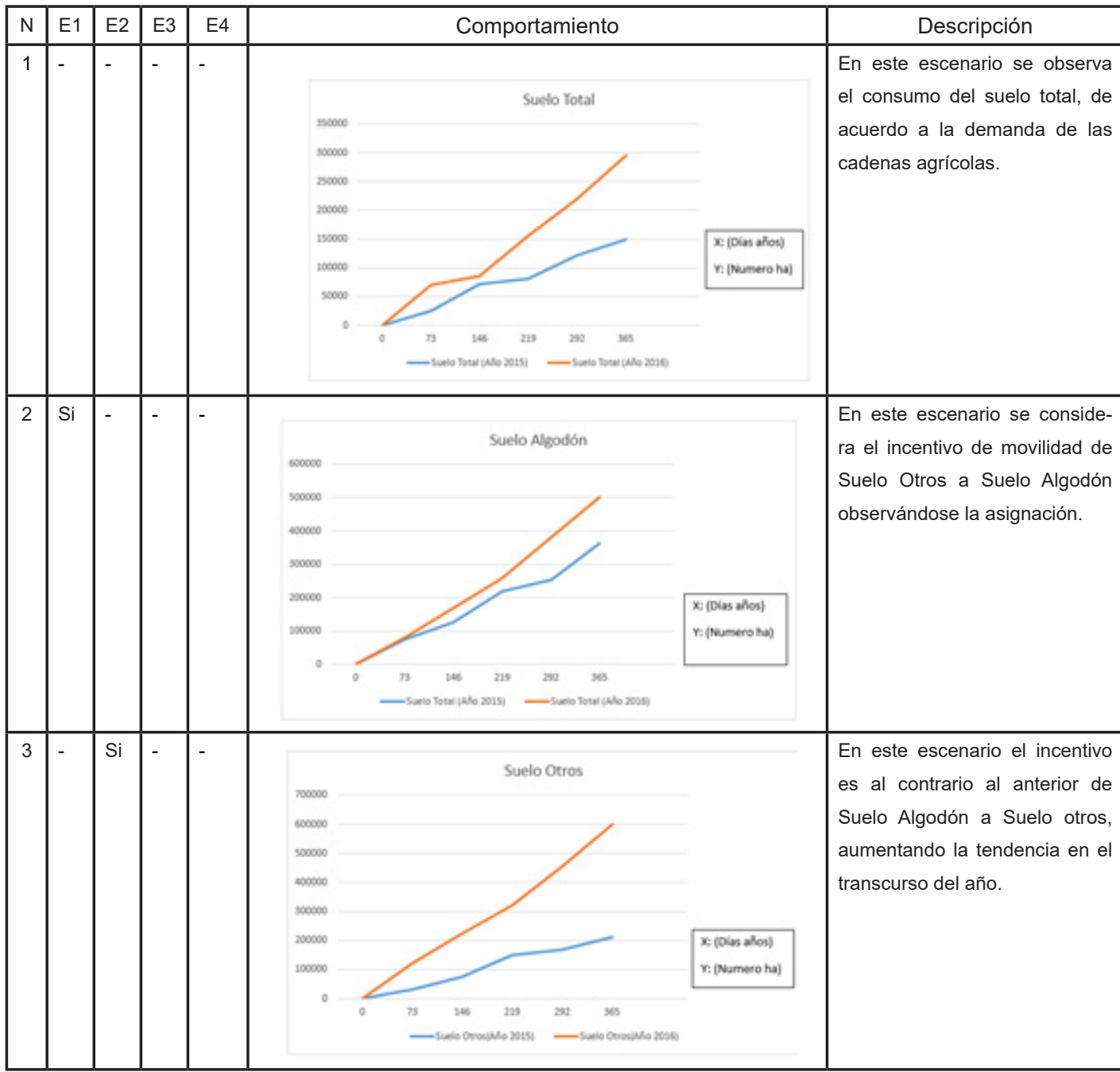




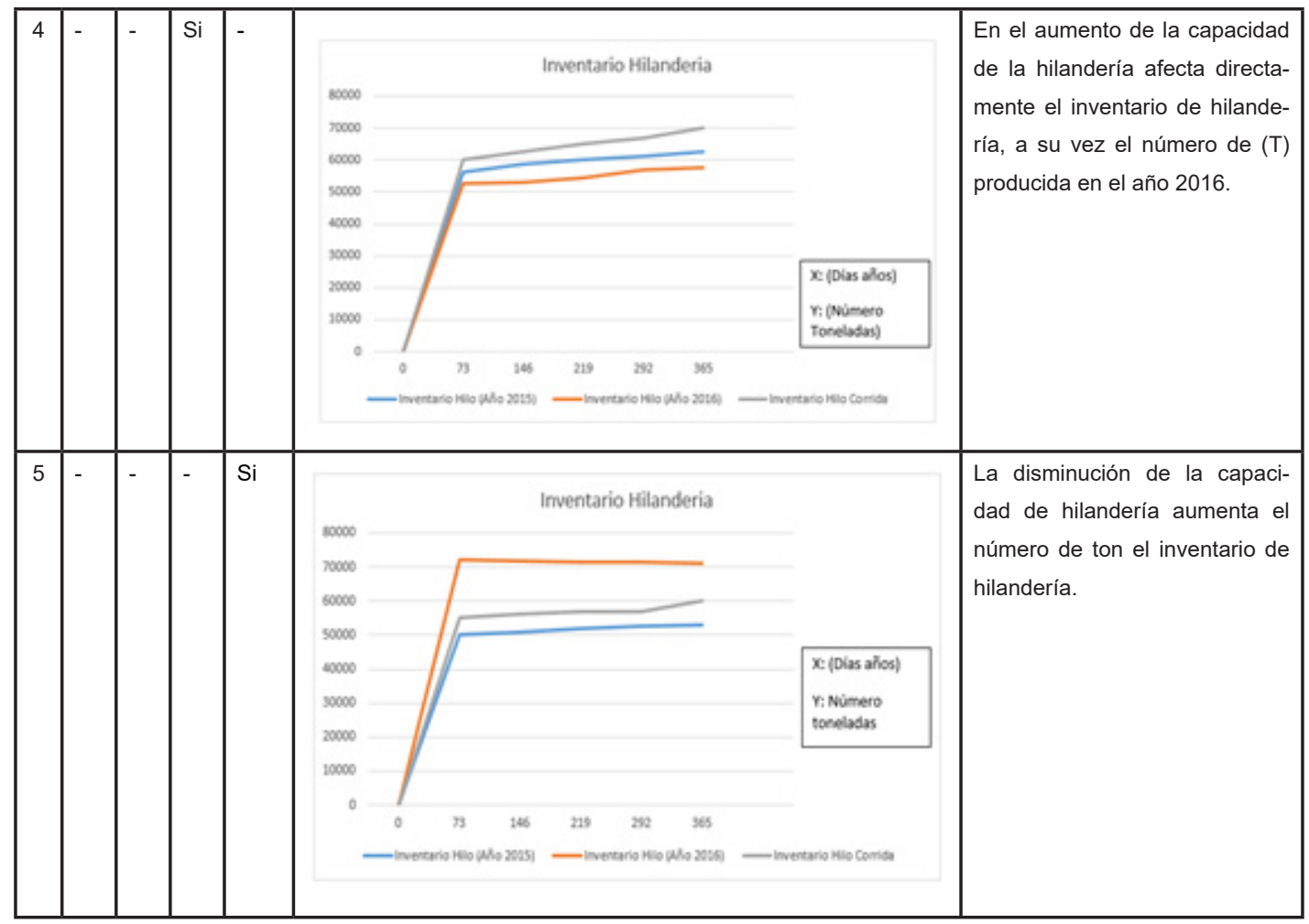

En la tabla 5, se evidencio que para los primeros meses el inventario del algodón desmotado en toneladas es alto, principalmente a que la producción del algodón en los últimos meses del año es la más alta, posterior en los últimos meses ya no se tiene inventario en algodón desmotado debido a que la producción es enviada a los inventarios de hilandería para continuar el proceso en la cadena.

Tabla 5. Inventario algodón desmotado. (Tabla de Tiempo)

\begin{tabular}{|c|c|c|}
\hline Tiempo (Meses) & Inventario Algodón Desmotado & Inventario Algodón Desmotado \\
\hline 0 & Año 2016 & 8547 \\
\hline 1 & Año 2015 & 28798 \\
\hline 2 & Current & 30748,66 \\
\hline 3 & & 26274,86 \\
\hline 4 & & 20405,19 \\
\hline 5 & & 14085,15 \\
\hline 6 & & 9727,43 \\
\hline 7 & & 6729,48 \\
\hline 8 & & 4669,56 \\
\hline 9 & & 3254,64 \\
\hline 10 & & 2282,27 \\
\hline 11 & & 1613,12 \\
\hline 12 & & 1151,61 \\
\hline
\end{tabular}


En la tabla 6, se observa el cambio de los últimos 6 meses del año en la capacidad de hilandería, esto directamente afectado por el incremento de la demanda y a su vez del aumento de la producción del algodón en las regiones costa e interior del país.

Tabla 6. Capacidad hilandería algodón (Tabla de tiempos)

\begin{tabular}{|c|c|c|}
\hline Tiempo (Meses) & Capacidad Hilandería & Capacidad Hilandería \\
\hline 0 & Año 2016 & 0,5 \\
\hline 1 & Año 2015 & 0,5 \\
\hline 2 & Current & 0,5 \\
\hline 3 & & 0,5 \\
\hline 4 & & 2,5 \\
\hline 5 & & 4,5 \\
\hline 6 & & 6,5 \\
\hline 7 & & 8,5 \\
\hline 8 & & 10,5 \\
\hline 9 & & 12,5 \\
\hline 10 & & 14,5 \\
\hline 11 & & 16,5 \\
\hline 12 & & 18,5 \\
\hline
\end{tabular}

En la tabla 7, se observa que para los primeros 2 meses el inventario hilandería en toneladas para la cadena del algodón es bajo, pues se considera que para los primeros meses del año la capacidad de hilandería es baja, debido a la poca producción de algodón en estos meses del año. A partir del 3 mes se nota un crecimiento constante, hasta la finalización del año en la producción de hilandería de cadena del algodón. Que de acuerdo a los procesos de la agro cadena estas toneladas se convierten en el producto final; en el eslabón confección (tejidos de punto, tejidos planos, telas) hasta un producto terminado como prendas de vestir, artículos deportivos, y otros.

Tabla 7. Inventario hilandería algodón (Tabla de Tiempos)

\begin{tabular}{|c|c|c|}
\hline Tiempo (Meses) & Inventario Hilandería Corridas: & Inventarios Hilandería \\
\hline 0 & Año 2016 & 8500 \\
\hline 1 & Año 2015 & 22175,1992 \\
\hline 2 & Current & 18528,4785 \\
\hline 3 & & 36075,4453 \\
\hline 4 & & 41946,5547 \\
\hline 5 & & 44044,6719 \\
\hline 6 & & 46046,4922 \\
\hline 7 & & 47763,8438 \\
\hline 8 & & 49220,6523 \\
\hline 9 & & 50459,8867 \\
\hline 10 & & 51521,1484 \\
\hline 11 & & 52437,332 \\
\hline 12 & & 53234,9297 \\
\hline
\end{tabular}

Es importante destacar que los resultados indicaron una descompensación en la cadena del algodón, esto principalmente al consumo o demanda que presenta actualmente, la demanda del algodón en estos últimos años ha ido en constante crecimiento y se ha solventado con un 
aumento en las importaciones.

Por otro lado anualmente el rendimiento en relación Tonelada/Hectárea sigue siendo muy favorable, el problema radica en el área de cultivo o cosecha que es cada año menor, esto trae como consecuencia menos total producido de algodón. El área cultivada en Colombia se divide en dos regiones la región interior y la región costa, teniendo un gran margen en la participación de la producción total la región costa con al menos un $70 \%$ de la producción, mientras un $30 \%$ para la región interior, según datos de Fabricato para los últimos años en toneladas, se tiene estimada una demanda de 74000 ton, hoy en día la producción en las hectáreas cultivadas de los últimos años no superaron las 32000 Hectáreas para una producción total de 24000 (T), creando un déficit en el sector en cuanto a la demanda y oferta. (Coltejer, 2013).

Otro aspecto relevante en Colombia es que las políticas nacionales para la asistencia directa para los productores de algodón bajaron drásticamente en estos últimos años haciendo más difícil la producción y posterior venta. En la temporada 2014/15, la asistencia directa a los productores de algodón en Colombia se estimó en USD 1617 dólares, para un promedio de 9 centavos la libra (inferior a los 40 centavos la libra respecto a 2013/14). Durante 2015/16, los pagos directos del gobierno disminuyeron aún más de USD 971 dólares, promediando 5 centavos la libra. Los pagos reales en pesos colombianos descendieron en un $42 \%$ durante 2015/16, pero los pagos equivalentes en dólares estadounidenses se redujeron en un $47 \%$ por la depreciación de la moneda nacional. (ICAC, 2017).

\section{CONCLUSIONES}

Dentro de la investigación se analizó que el sector algodonero no cuenta con un sistema logístico, que pueda determinar una mayor producción y facilite la comercialización de los productos, de igual manera se logró identificar que en el eslabón cultivo de la agro cadena analizada, es donde se presenta la falta de conocimiento de prácticas logísticas debido a que su producción y utilidades no alcanzan para cubrir los costos que incurren mantener un departamento o área dedicada a esta labor.

En relación con las regiones estudiadas los departamentos constituidos por la región central carece de una adecuada infraestructura logística que le permita mejorar el flujo de las mercancías para posicionarse como una región más competitiva frente a la región costa que al contar con los puertos marítimos cerca y a su vez por sus vías en marcha tienen una ventaja competitiva en tiempos y costos. De acuerdo a estas circunstancias la industria nacional no abastece el mercado, y para los cálculos de satisfacer la demanda el estimado en área cosechada de algodón debe aumentar al menos un $210 \%$, para cumplir con las toneladas del consumo nacional de algodón y no acudir al mercado internacional.

En general la agro cadena del algodón tiene problemas en el aspecto de producción, los costos en la región interior y costa presentan un alto costo debido principalmente a los alquileres en las tierras, los insumos, el manejo de plagas y enfermedades, y el abonamiento de las mismas, cabe destacar que el gremio del algodón persiste en Colombia debido a las agremiaciones gubernamentales que brindan los subsidios, pero la llegada de nuevos mercados, principalmente por los tratados de libre comercio, crearon un efecto contrario en el mercado nacional pues no se tiene una protección a la producción nacional de la agro cadena del algodón en Colombia. 


\section{REFERENCIAS}

ALARCON, D. R., OROZCO, D. L., and RIVERA, F. J. Análisis dinámico de la capacidad de respuesta de una cadena de suministros de productos tecnológicos, caso Samsung. Entramado, 2016, 12(2), pp. 254-275.

ANGERHOFER, B. J., and ANGELIDES, M. C. System dynamics modelling in supply chain management: research review. In: Proceedings of the 32nd conference on Winter Simulation, 2000, pp. 342-351.

ARROYO, P., VILLANUEVA, M., GAYTÁN, J., and GARCÍA, M. Simulación de la tasa de reciclaje de productos electrónicos: Un modelo de dinámica de sistemas para la red de logística inversa. Contaduría y Administración, 2012, 51(1), 9-41.

CAKRAVASTIA, A., and DIAWATI, L. Development of system dynamic model to diagnose the logistic chain performance of shipbuilding industry in Indonesia. In: Proceedings of the 17th International Conference of the System Dynamics Society, 1999.

COLTEJER. Fabricato Coltejer. [online]. 2013. [cited: 01-05-2017], Available in: <www.fabricato.com.co>.

CONABIO. Comisión Nacional para el Conocimiento y Uso de la Biodiversidad, Sistema de Información de Organismos Vivos Modificados (SIOVM), [online]. 2017. [cited: 15-04-2017], Available in: <http://www.conabio.gob.mx/conocimiento/bioseguridad/doctos/consulta_SIOVM.html>.

CONALGODON. Confederación Colombiana del Algodón, Informe Anual Estadístico del Sector Algodón, [online]. 2015. [cited: 15-03-2017], Available in: <http://conalgodon.com/estadisticas/>.

DANE. Departamento Administrativo Nacional de Estadística, [online]. 2007. [cited: 23-062017], Available in: <https://www.dane.gov.co/index.php/estadisticas-por-tema>.

FORRESTER, J. W. Industrial Dynamics, 1971. Cambridge/MIT Press.

HERRERA, M. M., and ORJUELA, J. A. Perspectiva de trazabilidad en la cadena de suministros de frutas: un enfoque desde la dinámica de sistemas. Ingeniería, 2014, 19(2), 63-84.

HOLWEG, M., BICHENO, J. Supply chain simulation - a tool for education, enhancement and endeavour. International Journal of Production Economics, 2002, 78(2), 175-189.

ICA. Instituto Colombiano Agropecuario, [online]. 2010. [cited: 01-01-2017], Available in: <http://www.sic.gov.co/recursos_user/documentos/promocion_competencia/Estudios_Economicos/ALGODON.pdf>.

ICAC. International Cotton Advisory Committee, Políticas de producción y comercio que afectan la industria del algodón, [online]. 2017. [cited: 01-06-2017], Available in: <https://www. icac.org/cotton_info/publications/statistics/stats_wtd/gm-s_2016.pdf>.

MARTÍNEZ, M., RODRÍGUEZ, C., GIL, M., and MORRIS, A. System dynamics in the simulation of the effect of knowledge management on the supply chain of corn agroindustry. Revista 
Técnica de la Facultad de Ingeniería Universidad del Zulia, 2013, 36(1), 20-31.

SIC. Superintendencia de Industria y Comercio, Cadena de la fibra de algodón para textiles y confecciones, [online]. 2010. [cited: 01-01-2017], Available in: <http://www.sic.gov.co/recursos_user/documentos/promocion_competencia/Estudios_Economicos/ALGODON.pdf>.

STERMAN, J. Modeling managerial behavior: misperceptions of feedback in a dynamic decision making experiment. Management Science, 1989, 35(3), 259-385. 